\title{
Integrative Therapie einer Patientin mit komplexen psychosomatischen Beschwerden
}

\author{
Frank Zimmermann-Viehoffa ${ }^{a}$ Bernd Bergander $^{a} \quad$ Karin Meissner $^{b} \quad$ Hans-Christian Deter $^{a}$ \\ a Abteilung für Psychosomatik und Psychotherapie, Charité Universitätsmedizin Berlin, Deutschland \\ ${ }^{\mathrm{b}}$ Institut für Medizinische Psychologie, Ludwig-Maximilians-Universität, München, Deutschland
}

\author{
Schlüsselwörter \\ Dyspepsie · Depression · Homöopathie . \\ Psychosomatik · Placebo
}

\section{Zusammenfassung}

Der Fall einer 76-jährigen Patientin mit komplexen psychosomatischen Beschwerden wird dargestellt und diskutiert. Die Patientin litt unter depressiver Verstimmung, Panikattacken, chronischen Schmerzen sowie unter Dyspepsie mit Übelkeit und Appetitverlust. Im Rahmen der psychosomatisch-integrativen Behandlung sprach die Patientin bezüglich der Dyspepsie-Symptomatik gut auf eine ergänzende homöopathische Behandlung an. Der therapeutische Stellenwert der Homöopathie im dargestellten Fall wird vor dem Hintergrund der aktuellen Placebo-Forschung diskutiert.

\section{Kasuistik}

Die 76-jährige Patientin wurde nach vorausgegangener Vorstellung in der interdisziplinären Schmerzkonferenz der Abteilung für Psychosomatik und Psychotherapie zugewiesen. Die Patientin klagte über starke Schmerzen im Lendenwirbelsäulenbereich mit Ausstrahlung in das rechte Bein. Eine operative Denervierung dreier lumbaler Facettengelenke 5 Monate zuvor hatte die Schmerzen weiter verstärkt. Die Schmerzstärke wurde aktuell auf einer 10-stufigen Skala im Mittel bei 8 angegeben.

Begleitend bestand seit etwa 18 Monaten eine zunehmende depressive Entwicklung, die durch Freudlosigkeit, innere Anspannung, körperliche Erschöpfbarkeit und Schlafstörungen gekennzeichnet war. Vor der stationären Aufnahme war es

\author{
Key Words \\ Dyspepsia - Depression · Homeopathy · \\ Psychosomatics P Placebo
}

\section{Summary \\ Integrative Therapy of a Patient with Complex Psycho- somatic Complaints}

We present the case of a 76-year-old female patient with complex psychosomatic complaints. The patient suffered from depression, panic attacks, chronic pain and dyspepsia with nausea and loss of appetite. In addition to the conventional psychosomatic care, the patient received individual homeopathic treatment for her dyspepsia, resulting in complete remission. The therapeutic value of homeopathy in the present case is discussed in the context of recent findings from placebo research.

zweimalig zum Auftreten von Panikattacken gekommen. Die Patientin beschrieb ein von der Magengrube ausgehendes «Kribbeln mit starker Unruhe» sowie ein «innerliches Zittern» mit Todesängsten. Die psychosomatischen Behandlungsdiagnosen lauteten mittelgradige depressive Episode bei rezidivierender depressiver Störung, Panikstörung sowie anhaltende somatoforme Schmerzstörung. Aufgrund einer therapieresistenten gastroösophagealen Refluxerkrankung war 6 Wochen vor Aufnahme eine operative Intervention (Fundoplicatio nach Nissen) erfolgt. Die postoperative Gastroskopie hatte reguläre Verhältnisse gezeigt. Während das Sodbrennen durch die Operation vollständig verschwunden war, litt die Patientin seither täglich unter starker Übelkeit mit Appetitverlust. Geruch oder Anblick von Speisen verstärkten die Symptomatik. In Folge hatte die Patientin bereits $10 \mathrm{~kg}$ an Gewicht verloren.

\begin{tabular}{ll}
\hline KARGER & $\odot$ 2008 S. Karger GmbH, Freiburg \\
Fax +4976145207 14 & Accessible online at: \\
$\begin{array}{l}\text { E-mail Information@Karger.de } \\
\text { www.karger.com }\end{array}$ & www.karger.com/fok
\end{tabular}

Dr. Frank Zimmermann-Viehoff

Abteilung für Psychosomatik und Psychotherapie

Charité Universitätsmedizin Berlin, Campus Benjamin Franklin

Hindenburgdamm 30, 12200 Berlin, Deutschland

Tel. +4930 8443996

E-mail frank.zimmermann@charite.de 
Die Eigenanamnese ergab ein vor 9 Jahren diagnostiziertes Mamma-Karzinom, welches durch Ablatio mammae und Chemotherapie vollständig zur Remission gebracht worden war. Weitere Vordiagnosen lauteten arterieller Hypertonus, Hypercholesterinämie, Hyperurikämie und Hypothyreose. Die tägliche Medikation bei Aufnahme bestand aus $60 \mathrm{mg}$ Urapidil, $600 \mathrm{mg}$ Eprosartan, $95 \mathrm{mg}$ Metoprolol, 37,5 mg Hydrochlorothiazid, $180 \mathrm{mg}$ Diltiazem, $8 \mathrm{mg}$ Molsidomin, $120 \mathrm{mg}$ Isosorbiddinitrat, $100 \mathrm{mg}$ Allopurinol, $100 \mu \mathrm{g}$ L-Thyroxin, $100 \mathrm{mg}$ Acetylsalicylsäure und $40 \mathrm{mg}$ Omeprazol. Ferner wurde ein transdermales Fentanylpflaster $(50 \mu \mathrm{g} / \mathrm{h})$ alle 3 Tage angewandt.

Die internistisch-neurologische Untersuchung bei Aufnahme zeigte außer einer Varikose der Unterschenkel einen regulären Status. Die klinisch-chemische Analyse zeigte eine Hypokaliämie (2,7 mmol/1, Normbereich: 3,4-5,2 mmol/l) sowie eine leichte Erhöhung der Harnsäure $(6,6 \mathrm{mg} / \mathrm{dl}$, Normbereich: 2,5-6,5 mg/dl), des C-reaktiven Proteins $(0,73 \mathrm{mg} / \mathrm{dl}$, Normbereich: $<0,5 \mathrm{mg} / \mathrm{dl}$ ) sowie des Gesamtcholesterins $(221 \mathrm{mg} / \mathrm{dl}$, Normbereich: $<200 \mathrm{mg} / \mathrm{dl}$ ). Alle übrigen Laborparameter lagen im Normbereich.

\section{Stationäre Therapie und Verlauf}

Die Patientin wurde über 25 Tage stationär behandelt. Während des Aufenthaltes nahm die Patientin regelmäßig an psychotherapeutischen Einzelgesprächen, psychodynamischer Gruppentherapie, Gestaltungstherapie und Konzentrativer Bewegungstherapie (einem körperorientierten Psychotherapieverfahren) teil. Begleitend wurden krankengymnastische und physikalisch-therapeutische Maßnahmen angewandt.

Die Hypokaliämie wurde durch Absetzen des Diuretikums (Hydrochlorothiazid) und temporäre orale Kalium-Substitution behoben. Die Nitrate (Molsidomin und Isosorbiddinitrat) wurden an Tag 5 bei fehlenden klinischen Hinweisen auf das Vorliegen einer koronaren Herzkrankheit und negativer Koronarangiographie vor 3 Jahren abgesetzt. An Tag 5 wurde eine antidepressive Therapie mit Mirtazapin eingeleitet (initial $15 \mathrm{mg}$, nach 5 Tagen Aufdosierung auf $30 \mathrm{mg}$ ), die von der Patientin gut toleriert wurde.

In den psychotherapeutischen Gesprächen berichtete die Patientin von traumatischen Erlebnissen während des 2. Weltkrieges. Frühe Erfahrungen von Todesangst und Ohnmacht hatten hier zu einer weiter bestehenden Angstbereitschaft geführt, die die Patientin während ihres Lebens überwiegend gut kompensieren konnte. Seit 13 Jahren lebe sie mit einem neuen Partner in einer gemeinsamen Wohnung. Diese Beziehung erlebe sie erstmals in ihrem Leben als unterstützend. Durch die zunehmenden körperlichen Beschwerden und manifesten Erkrankungen wurden frühe traumatische Ängste und Stresserfahrungen auf somatischer und seelischer Ebene erneut mobilisiert. Diese führten zu schwer fassbaren und unspezifischen Befindlichkeitsveränderungen, die das Selbst- wertgefühl tief erschütterten. Aufgrund der Befürchtung, dem 6 Jahre jüngeren Partner an Attraktivität und Vitalität nicht mehr zu genügen, kam es zu einer massiven Verunsicherung der Partnerschaft. Die hiermit verbundenen Konflikte und Affekte konnten psychotherapeutisch gut bearbeitet werden.

Die Dyspepsie-Symptomatik mit Übelkeit und Appetitverlust blieb hingegen zunächst unbeeinflusst. Eine probatorische Therapie mit einem Prokinetikum (Metoclopramid) erbrachte keine Besserung der Symptomatik und wurde daher nicht weitergeführt. Die Patientin nahm an Gewicht von $69 \mathrm{~kg}$ bei Aufnahme (BMI 28,7 kg/m²) auf 67,7 kg (BMI 28,2 kg/m²) an Tag $17 \mathrm{ab}$. Unter Beibehaltung der Omeprazol-Medikation wurde nun ergänzend eine individualisierende homöopathische Behandlung begonnen. Aufgrund des Leitsymptoms «Übelkeit und Ekel vor Speisen schon beim Denken an oder dem Geruch von Speisen» [1] fiel die Wahl des Arzneimittels auf Colchicum. Bestätigend fanden sich im Arzneimittelbild die Schmerzsymptomatik ( «Schmerzen in den Gelenken ... Bevorzugt erscheint die Kreuz- und Steißbeingegend» [2]) sowie der Gemütszustand der Patientin («Sehr ängstlich und schreckhaft, traurig und reizbar» [2]) gut abgebildet. Aufgrund der aus homöopathischer Sicht geschwächten Lebenskraft der Patientin und der umfangreichen konventionellen Medikation wurde eine Tiefpotenz in täglicher Wiederholung der Arzneimittelgabe verordnet. Ab Tag 17 erhielt die Patientin Colchicum D6 (Hersteller: Deutsche Homöopathie-Union DHU), 1mal täglich 1 Tablette. Innerhalb von 24 Stunden kam es hierauf zu einem vollständigen Sistieren der gastrointestinalen Symptomatik. Das Gewicht der Patientin steigerte sich bis zur Entlassung auf 68,5 kg (BMI 28,5 kg/m²). Die kontinuierliche Einnahme von Colchicum wurde bei Entlassung abgesetzt und eine weitere Einnahme bei Bedarf empfohlen. Gegen Ende der Behandlung hatte sich die Patientin auch bezüglich der anderen Beschwerden unter der kombinierten internistisch/psychosomatischen Therapie einschließlich homöopathischer Behandlungsaspekte bei einer ausgewählten Symptomatik deutlich stabilisiert. Die psychische Besserung spiegelte sich in der testpsychologischen Diagnostik wider. In der «Hospital Anxiety and Depression Sale» (HADS, [2]) hatte sich der Angst-Score von 10 auf 6 Punkte und der Depressions-Score von 8 auf 7 Punkte reduziert. Die Werte lagen somit bei Entlassung im subklinischen Bereich. Die Schmerzstärke war von 8 Punkten auf durchschnittlich 5 gesunken. Die Übelkeit war auch bei einem poststationären Gespräch 2 Wochen nach Entlassung nicht wieder aufgetreten; das Gewicht war stabil geblieben. Bei subjektiver Beschwerdefreiheit bezüglich der Dyspepsie war keine weitere Einnahme des homöopathischen Medikamentes erfolgt.

\section{Diskussion}

Der dargestellte Fall schildert die erfolgreiche psychosomatisch-integrative Therapie eines komplexen Krankheitsbildes. 
Im Folgenden soll der Stellenwert der Homöopathie im Rahmen der Gesamtbehandlung diskutiert werden. Die Indikation $\mathrm{zu}$ einem homöopathischen Behandlungsversuch wurde in der anfänglichen Therapieresistenz der Oberbauch-Symptomatik gegenüber psychotherapeutischen und medikamentösen Interventionen gesehen, wobei die Patientin nach der Aufnahme weiterhin an Gewicht verlor.

Anzunehmen ist, dass somatische (veränderte Anatomie durch die vorangehende Magenoperation, unerwünschte Medikamentenwirkungen) und psychische Faktoren (Übelkeit als Angst-Korrelat bzw. Appetitverlust im Rahmen des depressiven Syndroms) für die Symptomatik bedeutsam waren. Ebenso ist die Anti-Reflux-Operation aus homöopathischer Sicht als «Unterdrückung» interpretierbar. Obwohl der homöopathischen Behandlung ein promptes Sistieren der Beschwerden folgte, verdeutlicht der dargestellte Fall beispielhaft die Schwierigkeit der Beurteilung eines homöopathischen Therapieerfolges vor dem Hintergrund eines komplexen Krankheitsbildes sowie komplexer, parallel stattfindender Therapieansätze. Zweifellos hatten die antidepressive Pharmakotherapie sowie die psychotherapeutischen und physiotherapeutischen Behandlungen maßgeblich zum Behandlungserfolg beigetragen.

Für eine Wirksamkeit der homöopathischen Behandlung sprechen die folgenden Argumente:

1. Es bestand ein enger zeitlicher Zusammenhang zwischen Intervention und Besserung der Beschwerden.

2. Auf etablierte konventionelle Therapieversuche [3] mit Protonenpumpeninhibitor (Omeprazol) und Prokinetikum (Metoclopramid) hatte die Patientin zuvor nicht angesprochen.

3. Die Patientin führte die Besserung subjektiv auf das homöopathische Medikament zurück.

4. Zwischen dem Beschwerdebild der Patientin und dem Arzneimittelbild von Colchicum bestand ein hoher Grad der Ähnlichkeit (Homöopathizität).

In Anbetracht der hohen Placebo-Response-Rate bei funktionellen gastrointestinalen Störungen von etwa 40\% [4] ist jedoch eine unspezifische Wirkung nicht auszuschließen. Unter der Annahme einer Placebo-Wirkung können verschiedene Mechanismen zur Erklärung herangezogen werden [5]:

1. Die beobachtete Abnahme der Beschwerden entspricht einem Spontanverlauf bzw. einer Regression zum Mittelwert. Dies erscheint aus unserer Sicht in Anbetracht der Konstanz der Beschwerden über 6 Wochen und der prompten, über mindestens 3 Wochen andauernden Verbesserung unter homöopathischer Therapie eher unwahrscheinlich.

2. Die Wirkung ist durch klassische Konditionierung vermittelt. Dies setzte voraus, dass die Patientin zuvor positive Lernerfahrungen bezüglich einer Symptomverbesserung durch Medikamente gemacht hätte. Im dargestellten Fall hatte die Patientin jedoch auf verschiedene vorausgegangene Therapieversuche nicht angesprochen. Ebenso wenig hatte die Patientin Vorerfahrungen mit Homöopathie.

3. Die Wirkung ist über einen Suggestionsmechanismus vermittelt, der in der Patientin eine positive Erwartung bezüglich des Krankheitsverlaufes erzeugt. Eine starke suggestive Komponente war in der Behandlung der Patientin unzweifelhaft wirksam. Die homöopathische Intervention war eingebettet in eine tragfähige und vertrauensvolle Arzt-Patienten-Beziehung und psychotherapeutische Interventionen. Es ist daher auch denkbar, dass das homöopathische Medikament im Rahmen des therapeutischen Prozesses als Vehikel diente, welches die Loslösung von körperlichen Symptomen ohne Gesichtsverlust für die Patientin erleichterte. Forschungsergebnisse zeigen, dass es sich bei Placebo-Effekten nicht ausschließlich um subjektive, psychologische Phänomene handelt. Vielmehr lassen sich in vielen Fällen Veränderungen zentralnervöser, vegetativer, endokriner und immunologischer Funktionen nachweisen [6]. So ist beispielsweise der Placebo-Effekt bei Parkinson-Patienten über eine vermehrte endogene Dopamin-Ausschüttung vermittelt [7]. Auch die Magenmotilität, der in der Pathogenese der funktionellen Dyspepsie eine wichtige Bedeutung zukommt, lässt sich durch Placebo-Gabe messbar beeinflussen [8]. Die Ähnlichkeit solcher Wirkmechanismen zur homöopathischen Theorie einer «Aktivierung der Selbstheilungskräfte» ist auffällig [9]. Eine sichere Differenzierung von homöopathischer Wirkung und einer PlaceboAntwort erscheint daher anhand eines Einzelfalles praktisch unmöglich.

\section{Schlussfolgerung}

Der therapeutische Stellenwert der homöopathischen Intervention kann anhand des dargestellten Falles nicht sicher beurteilt werden. Die Patientin begrüßte jedoch das Angebot einer ergänzenden homöopathischen Behandlung und war dankbar für die Linderung der Beschwerden und den damit verbundenen Gewinn an Lebensqualität. Der dargestellte Fall zeigt aus unserer Sicht, dass komplementärmedizinische Verfahren wie Homöopathie gut in ein psychosomatisches Behandlungskonzept integrierbar sind und zum Wohle des Patienten genutzt werden können.

\section{Dank}

Ich danke der Patientin für ihr Einverständnis in die anonyme Veröffentlichung ihrer Fallgeschichte. Für die gute Zusammenarbeit danke ich ferner den Kollegen der kooperierenden Abteilungen. 


\section{Literatur}

1 Mezger J: Gesichtete homöopathische Arzneimittellehre, ed 12. Stuttgart, Haug, 2005.

2 Zigmond AS, Snaith RP: The Hospital Anxiety and Depression Scale. Acta Psychiatr Scand 1983;67: 361-70.

3 Moayyedi P, Soo S, Deeks J, Delaney B, Innes M, Forman D: Pharmacological interventions for nonulcer dyspepsia. Cochrane Database Syst Rev 2006; 18(4):CD001960.

4 Musial F, Klosterhalfen S, Enck P: Placebo responses in patients with gastrointestinal disorders. World J Gastroenterol 2007;13(25):3425-9.

5 Klosterhalfen S, Enck P: Psychobiology of the placebo response. Auton Neurosci 2006;125:94-9.

6 Benedetti F, Mayberg HS, Wager TD, Stohler CS Zubieta JK: Neurobiological mechanisms of the placebo effect. J Neurosci 2005;25(45):10390-402.

7 Meissner K, Distel H, Mitzdorf U: Evidence for placebo effects on physical but not on biochemical outcome parameters: a review of clinical trials. BMC Med 2007;19(5):3.

8 Meissner K, Gluender H, Mitzdorf U: Placebo effects on gastric slow wave frequency. Psychophysiology 2005;42:15-6(abstr).

9 Zimmermann-Viehoff F, Meissner K: Homeopathy and placebo - synonym, similar or different? Forsch Komplementmed 2007;14(4):247-8. 